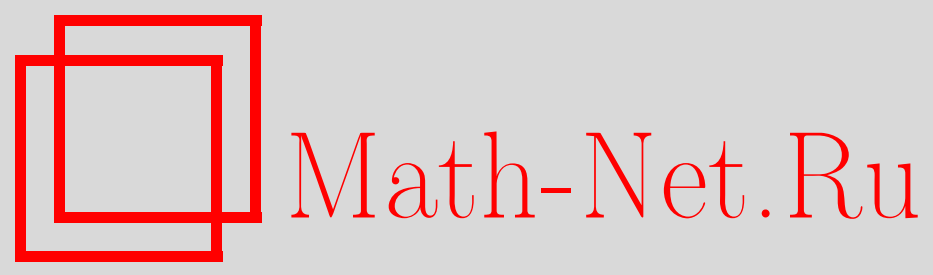

В. Н. Лебедев, Поиск и структура стационарных равновесий в циклических играх, Матем. заметки, 2000, том 67, выпуск 6, 913-921

DOI: https://doi.org/10.4213/mzm909

Использование Общероссийского математического портала Math-Net.Ru подразумевает, что вы прочитали и согласны с пользовательским соглашением http://www . mathnet.ru/rus/agreement

Параметры загрузки:

IP : 54.198 .55 .26

26 апреля 2023 г., $14: 20: 28$

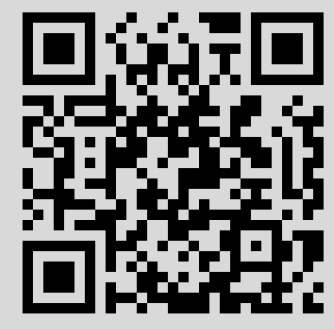




\title{
ПОИСК И СТРУКТУРА СТАЦИОНАРНЫХ РАВНОВЕСИЙ В ЦИКЛИЧЕСКИХ ИГРАХ
}

\author{
В.Н. Лебедев
}

\begin{abstract}
Доказывается существование оптимальных стационарных стратегий в цик лической игре, которая проходит по вершинам двудольного граффа до первого цикла, и платеж одного игрока другому есть сумма максимального и минимального локальных платежей этого цикла. Из полученного результата следует принадлежность задачи классу $\mathrm{NP} \cap \mathrm{co-NP}$, и для эргодических расширений матричных игр мы даем полиномиальный алгоритм нахождения оптимальных стратегий.
\end{abstract}

Библиография: 9 названий.

Введение. В [1] Л. С. Шепли дал следующее определение стохастической игры. Это игра двух лищ на множестве $N$ позиций. В позиции $k$ первый и второй игроки имеют $m_{k}$ и $n_{k}$ выборов соответственно. Игра считается неограниченной по длине. В позиции $k$ первый выбирает $i$-ю альтернативу, а второй игрок выбирает $j$-ю альтернативу. Тогда с вероятностью $p_{i j}^{k l}$ игра перейдет в позицию $l$, и первый игрок платит второму $c_{i j}^{k}, \mathrm{a}$ с вероятностью $s_{i j}^{k}>0$ игра остановится. Если при каждом $k$ либо $m_{k}=1$, либо $n_{k}=1$, то мы имеем игру с полной информацией. Для этого случая Шепли в [1] доказал существование стационарных оптимальных стратегий.

Используя этот результат, Д. Джильет [2] распространил его на случай,когда $s_{i j}^{k}=0$ при всех $i, j, k$ (в этом случае под вьпгрьшем понимается средний вьпгрьш за один шаг).

В [3] получено важное сведение детерминированных бесконечньх игр, где $p_{i j}^{k l}$ равно 0 или 1 при всех $i, j, k, l$, к конечным циклическим играм, где игра проходит по ребрам графа до первого цикла и платеж первого второму есть средняя величина полученного цикла.

В [4]-[7] изучались вопросы поиска оптимальных стратегий в циклических играх. В [4] показана геометрическая скорость определения цены игры с помощью динамического программирования Беллмана.

В [6] было замечено, что при наличии равновесия в стационарных стратегиях задача определения цены игры в заданной позиции относится к классу NP $\cap$ co-NP. Там же полностью описана структура оптимальных стратегий игроков с помощью алгоритма потенциальных преобразований. В [7] показано, что в худшем случае построенньй алгоритм экспоненциален от длины представления входной игровой сети, и отмечено, что полиномиального алгоритма решения таких игр автору не известно.

Пусть $G=(V ; E)=(B, A ; E)$ - конечньй, ориентированный, двудольньй граф с долями $A$ и $B$ без параллельных ребер. Ориентированное ребро с началом в вершине $v$ и концом в вершине $w$ обозначим $(v, w)$. Далее $E\left(V_{1}, V_{2}\right)$ обозначает множество ребер с 
началами в вершинах $V_{1}$ и конщами в вершинах $V_{2} ; E\left(V_{1}\right)$ обозначает множество ребер с началами в вершинах $V_{1} ; V\left(V_{1}\right)$ - множество конщевых вершин ребер с началами в вершинах $V_{1}$. Мы предполагаем беступиковость графа, т.е. множество $E(v)$ непусто для любой вершины $v$ графа.

Вершины $B$ (соответственно $A$ ) назьвают вершинами первого (соответственно второго) игроков. Рассматривается следующая позиционная антагонистическая игра двух лиц. Имеется фишка, которая в начальньй момент располагается в некоторой вершине $v_{0} \in V$. Игроки шаг за шагом передвигают фишку по ребрам графа по следуюшему правилу.

Если в текущий момент времени фишка находится в позиции $v \in B(v \in A)$, то первый (второй) выбирает некоторое ребро $(v, w) \in E(v)$ и передвигает ее в следующую вершину $w$. Предположим, что игра длится бесконечно долго, тогда фишка пройдет бесконечньй маршрут $T:=v_{0} \cdots v_{t} \cdots$. Пусть $c(T): T \rightarrow \mathbb{R}$ - платежный функционал пути в $G$. Тогда в разыгранной партии проигрыш первого, соответственно выигрыш второго, есть величина $c(T)$. Таким образом, первый минимизирует $c(T)$, а второй максимизирует это значение.

Основной вопрос этой статьи - изучение платежных функционалов $c(T)$, для которых имеется равновесие в стационарных стратегиях. Пусть каждому ребру $e \in E$ графа приписано целое число $c(e) \in \mathbb{Z}$ (целочисленная стоимостная функция на ребрах графа) и $T:=v_{0} \cdots v_{t} \cdots-$ маршрут игры. Хорошо изученным случаем (см. [5]-[8]) является средний предельный платежньй функционал:

$$
c(T)=\varlimsup_{t \rightarrow \infty} \frac{1}{t} \sum_{i=0}^{t-1} c\left(v_{i}, v_{i+1}\right) .
$$

В связи с отсутствием полиномиального алгоритма для этого случая функционала естественно ослабить ограничения на платежный функционал и рассмотреть позиционные средние. В этом случае выделены существенные классы игр с полиномиальным алгоритмом определения оптимальных стратегий. Так в [8] доказано наличие равновесия в стационарных стратегиях для максимального предельного платежного функционала для более общей постановки игровой задачи, там же представлен полиномиальный алгоритм решения таких игр.

В данной статье, используя результаты [9], изучается случай:

$$
c(T)=\varlimsup_{t \rightarrow \infty} c\left(v_{t}, v_{t+1}\right)+\underset{t \rightarrow \infty}{\lim } c\left(v_{t}, v_{t+1}\right) .
$$

(Если стратегии игроков стационарны, то это - просто сумма максимального $c(e)$ и минимального $c(e)$ ребер цикла, на который вьйдет игра. Для позиционного среднего в определении выше мы должны сумму разделить на два, тем не менее для сокращения записи коэффициент перед суммой будем опускать.)

В [9] рассмотрен частный случай результата данной работы, так называемые симметрические игры. Несмотря на применение близкого метода форсирования наилучшей позиции, доказательство индуктивного перехода существенно усложняется в лемме 1 настоящей работы, точно так же в общей постановке возникает более сложньй по структуре и по построению подграф-шлейф, позволяющий провести индуктивньй развал начальной игры. 
В настоящей работе мы доказьваем существование оптимальных стационарных стратегий и даем полиномиальный алгоритм их поиска в случае полного симметрического графа игры.

1. Теорема существования равновесия. Пусть $S_{1}, S_{2}$ - множества всех стратегий первого и второго игроков в заданной игре. Пусть $T\left(s_{1}, s_{2}, v_{0}\right)$ - маршрут из начальной вершины $v_{0}$, которьй соответствует стратегиям $s_{1} \in S_{1}$ и $s_{2} \in S_{2}$ игроков, и $c\left(s_{1}, s_{2}, v_{0}\right)$ - значение платежного функционала на этом маршруте. В дальнейшем нам потребуются определения стационарной стратегии и эргодического разбиения, которые можно найти в [9].

ТЕорема. Для каждой игры на графе $(B, A ; E)$ с иелочисленной стоимостной функиией $c: E \rightarrow \mathbb{Z}$ существуют стационарные стратегии $\beta$ и $\alpha$ первого и второго игроков такие, что для любой начальной вершины $v_{0}$ выполнено следующее седловое условие:

$$
\sup _{s_{2} \in S_{2}} c\left(\beta, s_{2}, v_{0}\right)=\inf _{s_{1} \in S_{1}} c\left(s_{1}, \alpha, v_{0}\right):=c\left(v_{0}\right)
$$

Величину $c\left(v_{0}\right)$ называют иеной игры в позиции $v_{0}$. Инфимум (супремум) берется по всем, необязательно стационарным, стратегиям игроков.

Далее подграф, порожденньй подмножеством вершин $W \subseteq V$, будем обозначать через $G(W)$.

В [5] показано, что задача “существует ли нетривиальное эргодическое разбиение в заданном графе?” является NP-полной проблемой.

Из определения эргодического разбиения следует

УТВЕРЖДЕНИЕ 1. Пусть $V_{1} u V_{2}$ - эргодическое разбиение $V$ в графе $G(V) u$ $V_{3}, V_{4}$ - эргодическое разбиение $V_{2}$ в подграфе $G\left(V_{2}\right)$. Тогда разбиение $V_{1} \cup V_{3}, V_{4}-$ әргодическое разбиение $V$ в графе $G(V)$.

ОПРЕДЕЛЕНИЕ 1. Граф $(B, A ; E(B, A) \cup E(A, B))$ с целочисленной стоимостной функцией $c: E \rightarrow \mathbb{Z}$ называется равновесным $c$ иеной $J$, если существуют две стационарные стратегии $\beta$ и $\alpha$ первого и второго игроков такие, что справедливы следующие условия:

1) цена всех простых циклов графа $(B, A ; \beta \cup E(A, B))$ не больше $J$;

2) цена всех простых циклов графа $(B, A ; E(B, A) \cup \alpha)$ не меньше $J$.

$\Gamma$ раф $(B, A ; \beta \cup E(A, B))$ кратко будем обозначать как $(V ; \beta)$, для второго графа соответствуюшее обозначение $-(V ; \alpha)$.

ОПРЕДЕЛЕНИЕ 2. Цена простого иикла $C:=v_{0} \cdots v_{k} v_{0}$ определяется как сумма максимального и минимального платежа ребер цикла: $\max _{e \in E} c(e)+\min _{e \in E} c(e)$, где $E-$ множество ребер цикла.

Легко видеть, что цена игры для любой начальной вершины $v_{0}$ равновесного графа есть $J$ и $\beta, \alpha$ - две оптимальные стационарные стратегии первого и второго игроков из теоремы.

Сформулированная вьше теорема непосредственно следует из лемм 1 и 2. 
Лемма 1. Пусть $(B, A ; E)$ - граф игры с иелочисленной стоимостной функиией $c: E \rightarrow \mathbb{Z}$ и J- произвольное челое число. Тогда существуют

(1) эргодическое разбиение $V_{1}, V_{2}$ (возможно $V_{1}=\varnothing$ или $\left.V_{2}=\varnothing\right)$

и две стационарные стратегии $\beta_{1}, \alpha_{2}$ для $G\left(V_{1}\right)$ и $G\left(V_{2}\right)$ соответственно такие, что справедливо следующее:

(2) чена всех простых ииклов графа $\left(V_{1} ; \beta_{1}\right)$ не больше $J-1$;

(3) чена всех простых ииклов графа $\left(V_{2} ; \alpha_{2}\right)$ не меньше $J$.

Обозначим $A_{1}:=V_{1} \cap A, B_{1}:=V_{1} \cap B$. Аналогично определим $A_{2}$ и $B_{2}$. Первый игрок гарантирует, что его потери составят не более $J-1$, если он применит стационарную стратегию $\beta_{1}$ в графе $G\left(V_{1}\right)$. Точно так же второй игрок обеспечит себе вьпгрьш не менее $J$, если применит стационарную стратегию $\alpha_{2}$ в графе $G\left(V_{2}\right)$.

ОПРЕДЕЛЕНИЕ 3. Шлейфом первого игрока, порожденньм множеством $V_{1} \subseteq V$ в графе $(V ; E)$, называется множество вершин $P$, полученное следующим процессом пометок (см. рис. 1, где черные (белые) вершины - позиции первого (второго) игрока).

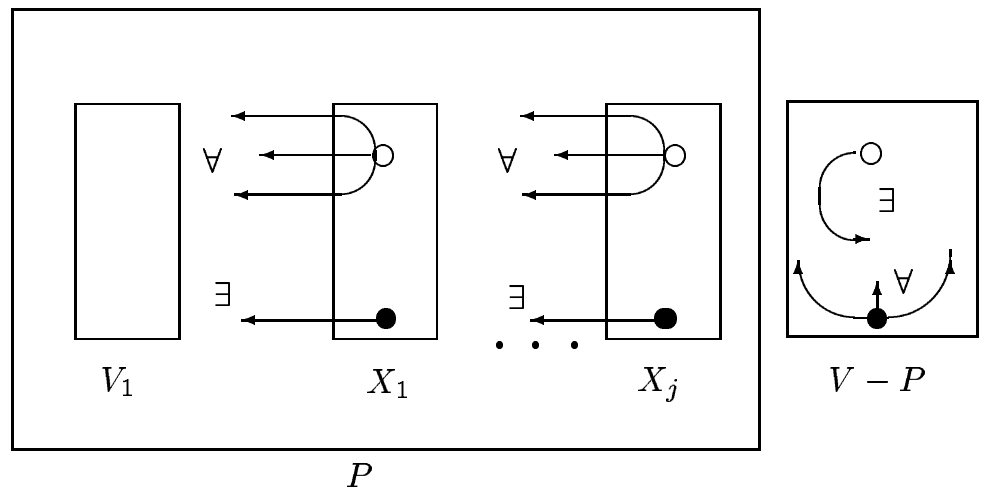

Рис. 1

Вначале помечаем множество $X_{0}=V_{1}$. Предположим, что уже помечены множества $X_{0}, \ldots, X_{j}$ - попарно не пересекающиеся непустые подмножества $V$. Берем $P_{j}:=X_{0} \cup$ $\cdots \cup X_{j}$. Определяем

$$
X_{j+1}:=\left\{v_{A}, v_{B} \in V-P_{j}: V\left(v_{A}\right) \subseteq P_{j}, V\left(v_{B}\right) \cap P_{j} \neq \varnothing\right\} .
$$

Определим стационарное ребро $\left(v_{B}, w\right)$ для каждой $v_{B} \in X_{j+1}, j \geqslant 0$, как одно ребро $\left(v_{B}, w\right)$, для которого $w \in P_{j}$. Множество стационарных ребер обозначим $\beta$. Если $X_{j+1}=\varnothing$, процесс нанесения пометок завершается и $P_{j}-$ результирующее множество $P$.

Пусть $P^{\prime}:=P-V_{1}$.

Конечное множество стационарных ребер $\beta$ назовем стационарной стратегией, порожденной множеством $V_{1}$.

Для второго игрока вводятся аналогичные понятия шлейфа и стационарной стратегии, порожденных множеством $V_{1}$ (см. рис. 2 , множество $V_{1}$ обозначено как $I$ ).

А именно, помечаем множество $X_{0}:=I$. Предположим, что мы уже пометили непересекаюшиеся непустые множества вершин $X_{0}, \ldots, X_{j}$. Обозначаем $L_{j}:=X_{0} \cup \cdots \cup X_{j}$. 



$L$

Рис. 2

Определяем $X_{j+1}$ как еще непомеченные вершины $v$ первого игрока, для которых $V(v) \subseteq L_{j}$, и вершины второго игрока, для которых $V(v) \cap L_{j} \neq \varnothing$, т.е.

$$
X_{j+1}:=\left\{v_{B}, v_{A} \in V-L_{j}: V\left(v_{B}\right) \subseteq L_{j}, V\left(v_{A}\right) \cap L_{j} \neq \varnothing\right\} .
$$

Для каждой вершины $v_{A} \in X_{j+1}$ определим стационарное ребро $\left(v_{A}, w\right)$ как одно ребро, для которого $w \in L_{j}$. Множество стационарных ребер обозначаем $\alpha$. Если $X_{j+1}=\varnothing$, процесс пометок заканчивается и $L:=L_{j}$ - результирующее множество.

ДокАЗАТЕЛЬСТво ЛЕммы 1. Лемма 1 будет доказана индукцией по числу $n$ ребер $E$ в графе игры $G(V ; E)$.

Для $n=2$ утверждение очевидно. Пусть утверждение доказано для каждого натурального $m, 2 \leqslant m \leqslant n$. Пусть число ребер в графе $G(V ; E)$ равно $n+1, J$ целое.

В доказательстве леммы 1 дважды используется следующее

УТВЕРЖДЕНИЕ 2. Пусть лемма 1 справедлива для всех графов с не более чем $n$ ребрами и пусть в графе с $n+1$ ребрами найдется разбиение $V_{1}, V_{2}, V_{1} \neq \varnothing$, вершин $V$ на непересекаюшиеся множсества $V_{1} \cap V_{2}=\varnothing$ такое, что выполнены следующие условия:

для $V_{1}$ справедливы условия эргодичности: граф $G\left(V_{1}\right)$ нетупиковый $и E\left(B_{1}\right.$, $\left.V_{2}\right)=\varnothing$

существует стационарная стратегия $\beta_{1}$ в графе $G\left(V_{1}\right)$ такая, что чена всех простых ииклов $\left(V_{1} ; \beta_{1}\right)$ не больие $J-1$.

Тогда лемма 1 справедлива для данного графа с $n+1$ ребрами.

ДоказАтельство. Рассмотрим разбиение $V_{1}, V_{2}$. Условия эргодического разбиения для вершин $V_{1}$ справедливы, но условия эргодичности для вершин $V_{2}$, возможно, не имеют места. Подправим множество $V_{2}$, чтобы получить эргодическое разбиение.

Рассмотрим шлейф первого игрока $P$ и стационарную стратегию $\beta$, которые порождены множеством $V_{1}$ (см. рис. 1$)$.

Тогда из условий утверждения 2 и процесса пометок (см. рис. 1) следует

(4) разбиение $P, V-P$ эргодическое;

(5) цена всех циклов графа $\left(P ; \beta_{1} \cup \beta\right)$ не больше $J-1$.

Обозначим $W:=V-P$.

В силу условия $V_{1} \neq \varnothing$ для графа $G(W)$ существуют эргодическое разбиение $V_{1}:=W_{1}$, $V_{2}:=W_{2}, W_{1} \cup W_{2}=W$, и две стационарные стратегии $\beta_{1}^{\prime}$ и $\alpha_{2}^{\prime}$ в $W_{1}$ и $W_{2}$ соответственно такие, что справедливы условия 1)-3) леммы 1. 
Тогда для всего графа верно:

1) цена всех циклов графа $\left(P \cup W_{1} ; \beta_{1} \cup \beta \cup \beta_{1}^{\prime}\right)$ не больше $J-1$ согласно условиям (4), (5), (2);

2 ) цена всех циклов графа $\left(W_{2} ; \alpha_{2}^{\prime}\right)$ не меньше $J$ по условию (3);

3) разбиение $P \cup W_{1}, W_{2}$ эргодическое по утверждению 1.

То есть разбиение $P \cup W_{1}, W_{2}$ и пара стационарных стратегий $\beta_{1} \cup \beta \cup \beta_{1}^{\prime}$ и $\alpha_{2}^{\prime}$ требуемые для графа с $n+1$ ребрами. Утверждение 2 доказано.

Для функции $c$ определим значения $M:=\max _{e \in E} c(e), m:=\min _{e \in E} c(e)$ и множество вершин $I:=\{v:$ существует $(v, w) \in E(v), c(v, w)=M\}$. Возможны две ситуации:

i) $M+m \geqslant J$

j) $M+m \leqslant J-1$.

i) Пусть $M+m \geqslant J$. Здесь возможны два случая:

ii) сушествует вершина $v^{\prime} \in I \cap B$ такая, что $\left|E\left(v^{\prime}\right)\right|>1$;

iii) для каждой вершины $v^{\prime} \in I$ выполнено свойство $v^{\prime} \in A$ или $v^{\prime} \in B,\left|E\left(v^{\prime}\right)\right|=1$.

В случае ii) рассмотрим граф $G^{\prime}\left(V, E^{\prime}\right)$, где $E^{\prime}:=E-\left(v^{\prime}, w^{\prime}\right), c\left(v^{\prime}, w^{\prime}\right)=M$. Согласно ii) граф $G^{\prime}$ беступиковьй, и по индуктивному предположению существуют эргодическое разбиение $V_{1}, V_{2}$ и две стационарные стратегии $\beta_{1}, \alpha_{2}$ такие, что цена всех циклов графа $\left(V_{1} ; \beta_{1}\right)$ не больше $J-1$, а цена всех циклов граффа $\left(V_{2} ; E^{\prime \prime}, \alpha_{2}\right)$ не меньше $J$. (Здесь $E^{\prime \prime}:=E\left(B_{2}, A_{2}\right)-\left(v^{\prime}, w\right)$.)

Если $V_{1}=\varnothing$, то цена всех простых циклов графа $\left(V_{2} ; \alpha_{2}\right)$ не меньше $J$ по предположениям i). Тогда эргодическое разбиение $V_{1}=\varnothing, V_{2}$ и стационарная стратегия $\alpha_{2}$ требуемые.

Пусть теперь $V_{1} \neq \varnothing$. Тогда разбиение $V_{1}, V_{2}$ и стратегия $\beta_{1}$ удовлетворяют условиям утверждения 2 , поэтому индуктивная посылка верна для начального графа. Это завершает рассмотрение случая іi).

В случае iii) рассмотрим шлейф $L$ и стационарную стратегию $\alpha$ второго игрока, которые порождены множеством вершин $I$ (см. рис. 2). Стационарную стратегию $\alpha$ доопределим в вершинах $I$ по правилу: для каждой вершины $v_{A} \in X_{0}$ стационарное ребро есть $\left(v_{A}, w\right): c\left(v_{A}, w\right)=M$.

Доопределенную стратегию обозначим $\alpha^{\prime}$.

Из процесса пометок легко видеть, что

(6) цена всех циклов $\left(L ; \alpha_{L}^{\prime}\right)$ не меньше $J$ (здесь $\left.\alpha_{L}^{\prime}:=\alpha^{\prime} \cap E(L, L)\right)$;

(7) $c(e)=M$ для всех ребер из множеств $E\left(L_{B}, V-L\right)$ и $\alpha^{\prime} \cap E\left(L_{A}, V-L\right)$;

(8) множество ребер $E\left(V_{A}-L, L\right)$ пусто.

Если $V=L$, то эргодическое разбиение $V_{1}=\varnothing, V_{2}=L$ и $\alpha^{\prime}$ требуемые по свойству 6). Пусть $V-L \neq \varnothing$. Граф $G(V-L)$ не имеет тупиковых вершин, поэтому в графе $G(V-L)$ по индукции существуют

(9) эргодическое разбиение $V_{1}, V_{2}$ такое, что $V_{1} \cup V_{2}=V-L$, и две стационарные стратегии $\beta_{1}, \alpha_{2}$ такие, что справедливо:

(10) цена всех простых циклов $\left(V_{1} ; \beta_{1}\right)$ не больше $J-1$;

(11) цена всех простых циклов $\left(V_{2} ; \alpha_{2}\right)$ не меньше $J$.

Если $V_{1}=\varnothing$, то эргодическое разбиение $V_{1}=\varnothing, V_{2} \cup L$ и стационарная стратегия $\alpha_{2} \cup \alpha^{\prime}$ искомые; так как цена любого цикла $v_{0}, \ldots, v_{k}, v_{0}$, где $v_{0}, \ldots, v_{k} \in V_{2}$, не 
меньше $J$ по свойству (11), цена любого цикла $v_{0}, \ldots, v_{k}, v_{0}$, где $v_{0}, \ldots, v_{k} \in L$, не меньше $J$ ввиду предположения (6) и цена каждого другого цикла $v_{0}, \ldots, v_{i}, v_{i+1}, \ldots, v_{0}$, где $v_{i} \in L, v_{i+1} \in V_{2}$, не меньше $J$ в силу $\left.(7), \mathrm{i}\right)$.

Поэтому остается случай $V_{1} \neq \varnothing$. Рассмотрим разбиение $V_{1}, V_{2} \cup L$. Условия эргодического разбиения для вершин $V_{1}$ справедливы в силу $(9),(8)$ и для графа $\left(V_{1} ; \beta_{1}\right)$ все циклы не больше $J-1$, поэтому применимо утверждение 2 , что заканчивает рассмотрение случая і).

ј) Случай $M+m \leqslant J-1$ легко сводится к случаю $M+m \geqslant J$. А именно, преобразуем функцию $c$ по правилу $c^{\prime}(v, w)=-c(v, w), J^{\prime}=-J+1$ и считаем, что первьй игрок максимизирует стоимость, в то время как второй минимизирует эту величину. Тогда для построенной игровой сети имеем случай $J^{\prime} \leqslant M^{\prime}+m^{\prime}$. По вьше приведенному доказательству существуют эргодическое разбиение $V_{1}, V_{2}$ и две стационарные стратегии $\beta_{1}, \alpha_{2}$ первого и второго игроков в графах $G\left(v_{1}\right)$ и $G\left(v_{2}\right)$ соответственно такие, что справедливы следующие утверждения:

1) цена всех циклов графра $\left(V_{1} ; \beta_{1}\right)$ не меньше $J^{\prime}$;

$2)$ цена всех циклов граффа $\left(V_{2} ; \alpha_{2}\right)$ не больше $J^{\prime}-1$.

Тогда эргодическое разбиение $V_{1}, V_{2}$ и две стационарные стратегии $\beta_{1}, \alpha_{2}$ для первого и второго игроков в $G\left(V_{1}\right)$ и $G\left(V_{2}\right)$ соответственно требуемые для $c(v, w)$ и $J$. Лемма 1 доказана.

Лемма 2. Для каждого графа $(V ; E)$ с иелочисленной стоимостной функиией $c: E \rightarrow \mathbb{Z}$ существуют эргодическое разбиение $V_{1} \cdots V_{k}$ и возрастающая последовательность челых чисел $J_{1}<\cdots<J_{k}$ такие, что граф $G\left(V_{i}\right)$ равновесный $c$ иеной $J_{i}, i=1, \ldots, k$.

Легко видеть, что цена игры для любой вершины $i$-гограффа $v \in V_{i}$ есть $J_{i}, i=1, \ldots, k$.

ДокАЗАтЕЛьство. Для $J=2 m$ эргодическое разбиение из леммы 1 есть $V_{1}=\varnothing$, $V_{2}=V$ и $\alpha_{2}-$ любая стационарная стратегия второго игрока. Для $J=2 M+1$ эргодическое разбиение из леммы 1 есть $V_{1}=V, V_{2}=\varnothing$ и $\beta_{1}$ - любая стационарная стратегия первого игрока.

Рассмотрим значение $J:=J_{1}$ наименьшее возможное, для которого разбиение из леммы 1 имеет вид $V_{1}, V_{2}^{\prime}$, где $V_{1} \neq \varnothing$, и существуют стратегии $\beta_{1}, \alpha_{2}^{\prime}$, для которых (1)-(3) справедливо.

Тогда для $J_{1}-1$ разбиение из леммы 1 есть $V_{1}=\varnothing, V_{2}=V$ и существует стационарная стратегия $\alpha$ второго игрока такая, что цена всех простых циклов графа $(V ; \alpha)$ не меньше $J_{1}-1$. Пусть $\alpha_{1}:=\alpha \cap E\left(V_{1}, V_{1}\right)$ (из-за того, что $V_{1}, V_{2}^{\prime}$ - эргодическое разбиение, $\alpha_{1}$ есть стационарная стратегия второго игрока в $\left.G\left(V_{1}\right)\right)$. Поэтому имеем

(12) $V_{1}, V_{2}^{\prime}$ - эргодическое разбиение, причем $V_{1} \neq \varnothing$;

(13) цена всех циклов графа $\left(V_{2}^{\prime} ; \alpha_{2}^{\prime}\right)$ не меньше $J_{1}$;

(14) цена всех циклов графа $\left(V_{1} ; \beta_{1}\right)$ не больше $J_{1}-1$;

(15) цена всех циклов графо $\left(V_{1} ; \alpha_{1}\right)$ не меньше $J_{1}-1$.

Тогда граф $G\left(V_{1}\right)$ равновесный с ценой $J_{1}-1$ в силу $(14),(15)$. Для графа $G\left(V_{2}^{\prime}\right)$ наименьшее возможное $J:=J_{2}$, для которого эргодическое разбиение $V_{2}, V_{3}^{\prime}, V_{2} \neq \varnothing$, есть $J_{2}>J_{1}$ в силу (13). Индукцией по числу вершин в графе $(V ; E)$ из приведенных вьше рассуждений следует, что имеется последовательность цельх чисел $J_{1}-1<J_{2}-1<\cdots<$ 
$J_{k}-1$, эргодическое разбиение $V_{1}, V_{2}, \ldots, V_{k}$ вершин $V$ и множество стационарных стратегий $\beta_{1}, \beta_{2}, \ldots, \beta_{k}, \alpha_{1}, \alpha_{2}, \ldots, \alpha_{k}$ первого и второго игроков в графах $G\left(V_{1}\right), \ldots, G\left(V_{k}\right)$ соответственно такие, что цена всех простых циклов графа $\left(V_{i} ; \beta_{i}\right)$ не больше $J_{i}-1$ и цена всех простых циклов графа $\left(V_{i} ; \alpha_{i}\right)$ не меньше $J_{i}-1, i=1, \ldots, k$. Тогда граф $G\left(V_{i}\right)$ равновесньй с ценой $J_{i}-1$ и $\beta_{1} \cup \cdots \cup \beta_{k}, \alpha_{1} \cup \cdots \cup \alpha_{k}$ - оптимальные стационарные стратегии первого и второго игроков в игре $(V ; E)$. Лемма 2 доказана.

Теорема следует из леммы 2 непосредственно (см. [9]). Это завершает доказательство теоремы.

2. Сложность вычисления оптимальных стратегий. Из теоремы следует, что задача "верно или нет, что цена игры в данной начальной вершине $v$ не превосходит данного числа $p$ ?" относится к классу NP $\cap$ co-NP.

Однако, из доказательства теоремы можно построить лишь экспоненциальньй алгоритм, а полиномиальньй алгоритм решения данной задачи автору не известен. Сейчас будет представлен полиномиальньй алгоритм для так называемых эргодических расширений матричньх игр.

Пусть дан полньй граф $(A, B ; E)$ такой, что для каждых $v \in A, w \in B:(v, w) \in E$, $(w, v) \in E$. Стоимостная функция симметрична $c(v, w)=c(w, v)$.

Лемма 3. Любой полный граф игры $(A, B ; E)$ с произвольной челочисленной симметрической стоимостной функиией с равновесен, т.е. существуют иелое число $J$ и две стационарные стратегии $\beta, \alpha$ первого и второго игроков такие, что выполнены следующие соотношения:

1) чена всех простых ииклов графа $(V ; \beta)$ не больше $J$;

2) чена всех простых ииклов графа $(V ; \alpha)$ не меньше $J$.

ДокАЗАТЕЛЬСтво. Лемма 3 является непосредственным следствием леммы 2, так как в случаеполного двудольного графа любое эргодическое разбиение может состоять только из одного блока.

Мы повторим доказательство с небольшими изменениями, чтобы получить полиномиальный алгоритм для рассматриваемого случая. Сначала будет доказана

Лемма 4. Для полного графа $(A, B ; E)$ с симметрической иелочисленной стоимостной функиией с и для каждого иелого $J$ имеет место один из следующих двух случаев:

1) существует стационарная стратегия $\alpha$ второго игрока такая, что чена всех простых ииклов графа $(V ; \alpha)$ не меньше $J$;

2) существует стационарная стратегия $\beta$ первого игрока такая, что иена всех простых ииклов графа $(V ; \beta)$ не больие $J-1$.

ДокАЗАТЕЛЬСтво. Лемма 4 будет доказана индукцией по числу $n$ вершин $|V|$ в графе игры $(V ; E)$. Для случая $|A|=1$ или $|B|=1$ утверждение очевидно.

Пусть утверждение доказано для $n \geqslant 2$. Пусть число вершин $|V|=n+1$ и $|A| \geqslant 2$, $|B| \geqslant 2, J$-произвольное целое число. Для функции $c$ определим следующие величины: $M:=\max _{e \in E} c(e), m:=\min _{e \in E} c(e)$. Рассмотрим две возможности:

i) $M+m \geqslant J$

j) $M+m<J$. 
i) Рассмотрим вершину $v \in A$ такую, что $c(v, w)=M,(v, w) \in E(v)$ (такая вершина существует в силу симметричности функции). Рассмотрим граф $G^{\prime}:=G(V-v)$. Для этого графа по предположению индукции имеют место случи:

$\left.1^{\prime}\right)$ существует стационарная стратегия $\alpha^{\prime}$ такая, что цена всех простых циклов графа $\left(V-v ; \alpha^{\prime}\right)$ не меньше $J$;

$\left.2^{\prime}\right)$ существует стационарная стратегия $\beta^{\prime}$ такая, что цена всех простых циклов графа $\left(V-v ; \beta^{\prime}\right)$ не больше $J-1$.

Если вьполнено $\left.1^{\prime}\right)$, то тогда для начального графа имеем случай 1$)$ для стационарной стратегии $\alpha^{\prime} \cup(v, w)$ второго игрока, так как цена всех простых циклов графа $\left(V ; \alpha^{\prime} \cup\right.$ $(v, w))$ не меньше $J$ в силу предположений $\left.\left.1^{\prime}\right), \mathbf{i}\right)$.

Если вьполнено $2^{\prime}$ ), то для начального графа имеем случай 2 ), так как цена всех простых циклов графа $\left(V ; \beta^{\prime}\right)$ не больше $J-1$ по предположению $\left.2^{\prime}\right)$.

Случай ј) рассматривается аналогично. Лемма 4 доказана.

Из вьше приведенного доказательства следует алгоритм нахождения стратегий $\alpha$ и $\beta$ со сложностью $f(n) \leqslant f(n-1)+a n^{2}$, т.е. $f(n)=O\left(n^{3}\right)$ (a- некоторая константа).

Чтобы доказать лемму 3 , рассмотрим наименьшее возможное значение $J$ такое, что имеет место случай 2 ) леммы 4 . Тогда для $J-1$ мы имеем случай 1 ) леммы 4 . Таким образом, $J-1$ и две стационарные стратегии $\beta$ и $\alpha$ искомые в лемме 3 . Цену игры $J$ естественно определять дихотомией. Поэтому общее число элементарных операций алгоритма оценивается как $O\left(n^{3} \log _{2}(M-m+2)\right)$.

\section{СПИСОК ЦИТИРОВАННОЙ ЛИТЕРАТУРЫ}

[1] Shapley L. S. Stochastic games // Proc. Nat. Acad. Sci. USA. 1953. V. 39. P. 1095-1100.

[2] Gillette D. Stochastic games with zero stop probabilities // Ann. Math. Stud. 1957. V. 39. P. 178-187.

[3] Кифер Ю. И. Оптимальное поведение в играх с неограниченной последовательностью ходов // Теория вероятн. и ее примен. 1969. Т. 14. № 2. С. 284-291.

[4] Federgruen A., Schweitzer P. Contraction mappings underlying undiscounted Markov decision problems // J. Math. Anal. Appl. 1978. V. 65. P. 711-730.

[5] Ehrenfeucht A., Mycielski J. Positional strategies for mean payoff games // Intern. J. Game Theory. 1979. V. 8. № 2. P. 109-113.

[6] Гурвич В.А., Карзанов А. В., Хачиян Л.Г. Циклические игры и нахождение минимаксных средних циклов в ориентированных граф̆ах // ЖВМиМФ. 1988. Т. 28. №9. C. $1407-1417$.

[7] Лебедев В.Н. Алгоритмы оптимизации стационарных управлений в дискретных динамических системах. Дисс. ... к.ф.-м.н. М.: МФТИ, 1990.

[8] Karzanov A. V., Lebedev V. N. Cyclical games with prohibitions // Math. Programming. 1993. V. 60. P. 277-293.

[9] Лебедев В. Н. О новом равновесии в циклических играх // Дискретная матем. 1997. Т. 9. № 4. C. $96-99$. 